\title{
INTRODUCTION \\ The treatment of cerebral aneurysms: flow diversion and beyond
}

\author{
Adam S. Arthur, MD, MPH, ${ }^{1}$ Philipp Taussky, MD, ${ }^{2}$ Min S. Park, MD, ${ }^{2}$ Michael F. Stiefel, MD, ${ }^{3}$ and \\ Robert H. Rosenwasser, MD4
}

1Department of Neurosurgery, Semmes Murphey Neurologic and Spine Clinic, University of Tennessee Health Science Center, Memphis, Tennessee; ${ }^{2}$ Department of Neurosurgery, University of Utah, Salt Lake City, Utah; ${ }^{3}$ Capital Institute for Neurosciences, Trenton, New Jersey; and ${ }^{4}$ Department of Neurosurgery, Thomas Jefferson University, Philadelphia, Pennsylvania

\begin{abstract}
Be not the first by whom the new are tried, nor yet the last to lay the old aside.
\end{abstract}

\section{- Alexander Pope, quoted by Sir William Osler}

As new technologies become available, physicians and patients must decide how and when these technologies should best be used. Technology has driven successive generations of aneurysm treatment modalities from the beginning. These treatments have evolved considerably since Moniz performed the first cerebral angiogram in 1927 and Dandy clipped the first intracranial aneurysm in 1937. Surgical techniques have advanced from more indirect aneurysm occlusion treatments, such as Hunterian ligation and wrapping of aneurysms, to modern-day microsurgical clipping as pioneered by Yaşargil. Coil technology has been improved by advances in detachment mechanisms, stretch resistance, and adjunctive devices. With the introduction of flow diversion, a new paradigm in aneurysm treatment has arrived on the scene. The past several years have brought significant improvements in the technology, techniques, and understanding of this paradigm.

In the US only one flow diverter (the Pipeline embolization device) has been approved to date, and the indications for usage apply to only a small fraction of patients with aneurysms. Other flow diverters are in use in other parts of the world and may soon be available in the US as well. Additionally, the use of flow diversion in practice has rapidly expanded to include many other patients with aneurysms in a variety of clinical scenarios. This has created a knowl- edge gap in which neurointerventionalists are challenged to keep up with the options available to patients and the best practices for applying flow diversion strategies.

In this issue of Neurosurgical Focus, a variety of issues related to using flow diversion to treat cerebral aneurysms are explored. These topics include the cost-effectiveness, the use of platelet testing, complication avoidance, and vision outcomes when using flow diversion to treat paraclinoid aneurysms. Special-use cases reviewed include transcirculation deployment, iatrogenic dissections, tandem aneurysms, blister aneurysms, the retreatment of previously treated aneurysms, aneurysms associated with arteriovenous malformations, and off-label uses in general. As flow diversion becomes more widely applied it is hoped that we can understand how and in what circumstances it can best be used. This understanding should help to make the treatment of cerebral aneurysms as safe and effective as possible.

https://thejns.org/doi/abs/10.3171/2017.3.FOCUS17174

\section{Disclosures}

Dr. Arthur is a consultant for Codman, Medtronic, MicroVention, Penumbra, Siemens, and Stryker; receives research support from MicroVention and Siemens; and holds stock in Bendit, Cerebrotech, Serenity, and Synchron. Dr. Stiefel is a consultant for Penumbra, Medtronic, Codman, and Stryker. Dr. Taussky is a consultant for Medtronic. 